\title{
Approach of Image Processing in Diagnosis and Medication of Fungal Infections in Pet Animals
}

\author{
Aarti Chugh, Priyanka Makkar, Sourabh Aggarwal, Shriyansh Sharma, Yashwant Kumar Singh
}

\begin{abstract}
The focus of this paper is to design and develop the Artificial Intelligence and Machine Learning based system which detects the fungal infection on a pet animal (especially Dog and Cat), and provide the treatment for it. It also provides the causes, Symptoms, Prevention for it. The proposed system integrates on Machine Learning algorithm trained on disease dataset, it first detects the species of pet animal by capturing the image of fungal infection of pet by using camera or by uploading the image in the system from the file explorer, and then provide the diagnosis about the fungal disease and its medication and treatment also. Although our system will verify the scan image for determining the diseases in canine. Diagnosing the Fungal Infection on pet animal quickly and accurately has the economic effectiveness.
\end{abstract}

KEYWORDS- Disease Diagnosis Treatment Animal Pet Image Dataset Machine Learning Image processing.

\section{INTRODUCTION}

The pet animals may sometimes get suffered to fungal skin infections due to many reasons [1] [2] like scratching of their skin with their paw or getting in touch with some infected object also the skin of pet animals is sensitive in most cases so that they occupy the infection more rapidly than of street animals. And if some animal has got suffered with the infection so its owner has to take him to the hospital and then the doctors have to test the infection and wait for

\section{Manuscript received July 16, 2020}

Aarti Chugh, Assistant Professor, ASET, Amity University Haryana, Gurugram, India (e-mail: achugh@ggn.amity.edu)

Priyanka Makkar, Assistant Professor, ASET, Amity University Haryana, Gurugram, India

Sourabh Aggarwal, Student, ASET, Amity University Haryana, Gurugram, India

Shriyansh Sharma, Student, ASET, Amity University Haryana, Gurugram, India

Yashwant Kumar Singh, Student, ASET, Amity University Haryana, Gurugram, India reports to come and till then the pet may have to suffer and feel self-irritated. Our System will help the veterinarians to quickly and accurately diagnose the infection by analysing the scanned or uploaded image of infection on pet. Also, sometimes there may be a situation when there is not any veterinary hospital nearby and pet is getting irritated with the infection so badly, but there is a medical shop nearby but how do the owner know which medicine he has to bring, so in this case our system works, as the owner himself can diagnose the infection and its treatment by using the our system, and bring the medicine to his pet. The system is basically a web-based application which will run on browser without connecting to internet after its installation in the computer by connecting with its own server in the installed file. The system will work on the concept of Machine Learning by using TensorFlow and Open Source Computer Vision (Open CV) [3] to track the movement of camera, detect the image and recognize the infection. Bootstrap, CSS, Python, Tkinter are also used for making the interface and templates of system [4].

\section{RELATED WORK}

In Literature image Processing for detecting infections is reviewed by many papers, however there is not any study on detection, diagnosis, treatment(medication) altogether of fungal infection in pet animals. So, in our project we have tried to overcome this research gap by making the diagnosis and treatment detector of fungal infections of pet animals. N. Singh et. al. proposed the technologies like Image Processing, ANN, Data Mining. [5]. Niko Quiskamp et.al proposed the open-source image-processing framework, and uses OpenCV for the implementation. Also developed a GUI to load image data and then visualize the results.[6]. Keyvan Asefpour et.al. proposed the use of CCD Digital camera, personal computer and thermal camera for their system functioning.[7].Yogesh Joshi proposed comparison of different images to get the accurate results. [8]. For proposed system we use the open $\mathrm{cv}$, image pre-processing, artificial neural Networks, use the Camera for capturing the live real time image of the infection in an option to upload the previously captured image, use more than one image of every infection by taking the inspiration from all the researches reviewed.

\section{Methodology}

For the Proposed System, we use the Artificial Intelligence for detecting the captured or uploaded image of a pet fungal 
infection. The system uses the Neural network algorithms of TensorFlow and CNN (Convolutional Neural Networks) [9] and Algorithm Library of Open CV [10] which, first detects the species of the Pet and then provides the name of Infection and diagnosis to that infection. The Frame work and template formations are done by using Python, HTML, Bootstrap CSS Tools, the templates contains the options of Capturing, Uploading, Detecting Images, and a pane for showing results. IP Webcam camera application is used to capture image of Infection from phone camera. Machine learning for infection type detection is used in this system, the dataset attached to the system takes the image of the pet. The image converts into a byte array of greyscale, for image processing to detect infected areas using Open source computer vision. The machine learning model detection [11] classifies the category of the infected area. The image converts in two ways: firstly, using grayscale (the image converts into black and white and results in an array) \& secondly, through RGB Values (the coloured [12] image uses the RGB value of colour combination ranging from 0 to 255 .

\section{A. Capture Infection Sample or Select Infection Sample}

User can Capture the image of infection by using IP Webcam Camera application after connecting it to the system, by choosing Capture infection sample option on template of system or User can save the clicked image of infection in the file explorer and upload the image from the file explorer of device on which the system is running.

\section{B. Detection of Animal}

The algorithm for detecting fungal infection now process the captured or uploaded image to classify the species of pet animal. User have to click on Check Animal option on screen.

\section{Check Infection}

After the detection of animal, the system will run image processing to detect the type of Fungal Infection and give the results of treatment, medication, symptoms, cause.

\section{RESULT}

The results are as follows:

Fig.1-4 Shows the infection diagnosis and treatment in dog: As we see after the ip webcam captures the pet image or the image is selected from internal storage of computer, the analyzer analyzes that image for finding species of pet first (Fig.1) and the result shows that the species is dog (Fig.2). And then it analyzes this image for detecting the infection from which the pet is suffering and gives the name of infection and the result shows the disease is Aspergillosis (Fig.3). Afterwards the system gives the full diagnosis, way of treatment for that disease (Fig .4).

Fig.5-8 Shows Infection diagnosis and Treatment in Cat. As we see after the ip webcam captures the pet image or the image is selected from internal storage of computer, the analyzer analyzes that image for finding species of pet first (Fig.5), and the result shows that the species is cat (Fig.6). And then it analyzes this image for detecting the infection from which the pet is suffering and gives the name of infection, and the result shows that the disease is Aspergillosis (Fig.7). Afterwards the system gives the full diagnosis, way of treatment for that disease (Fig .8).

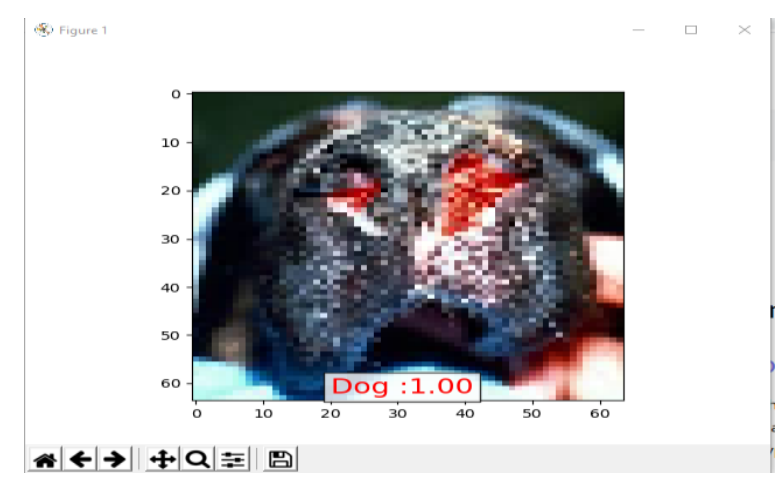

Fig. 1: Analysing the Species of Pet.
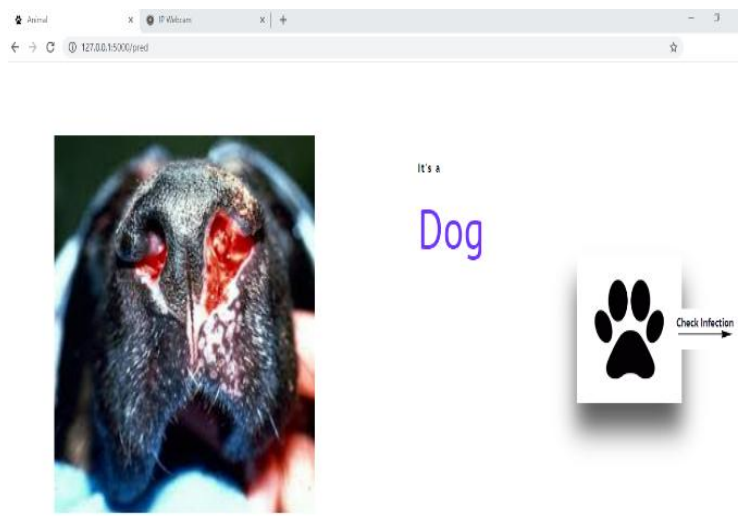

Fig. 2: Species analysed and Diagnosing infection.
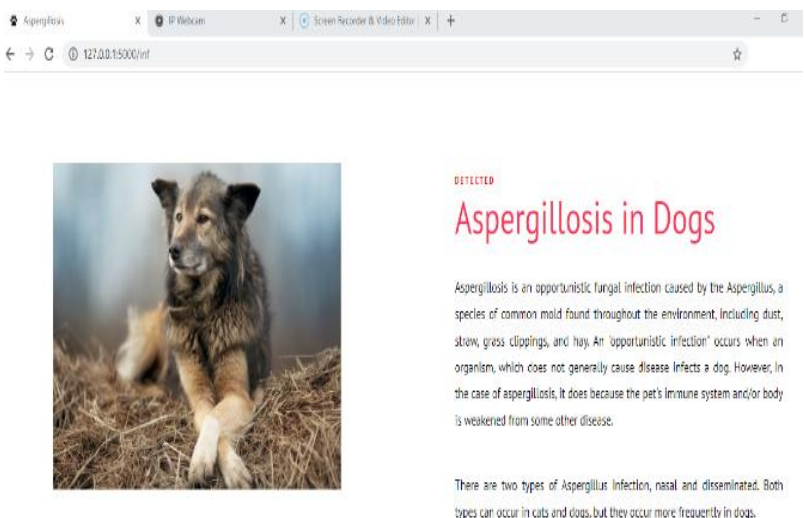

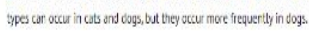

Fig.3: Infection Diagnosed.

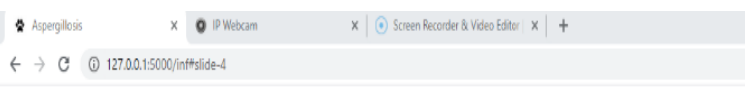

Treatment

Treatment varies depending on whether the disease is nasal or disseminated. The primary choice of treatment for dogs with nasal aspergitlosis is the administration of an antifungal drug directly into the patient's nose and nasal passages, while the patient is under anesthesia. Disseminated cases in dogs are diffecult to treat and rarely cured. Antifungal drugs are generally given to treat symptoms, and may cure the condition.

Fig. 4: Treatment of Infection shown. 


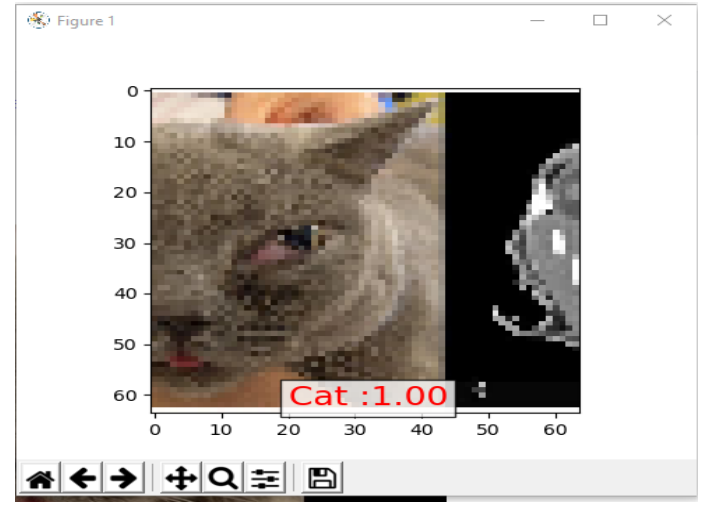

Fig.5: Analysing the Species of Pet.

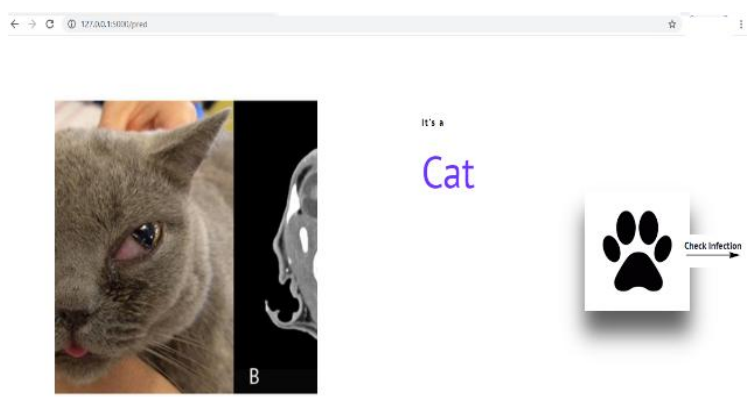

Fig.6: Species analysed and Diagnosing infection

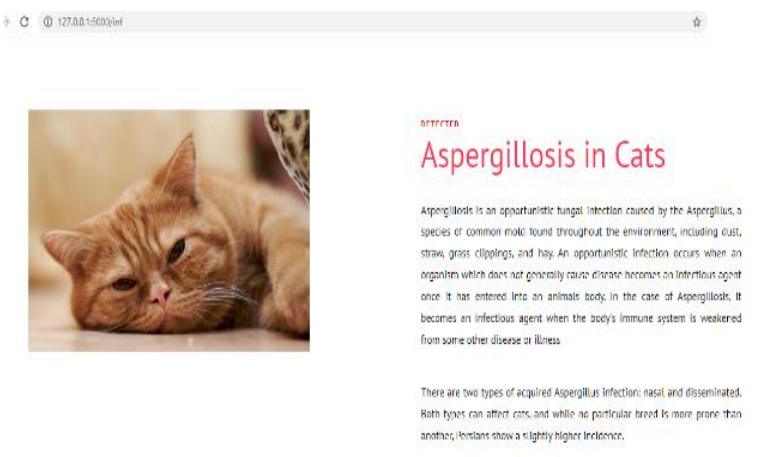

Fig.7: Infection Diagnosed.

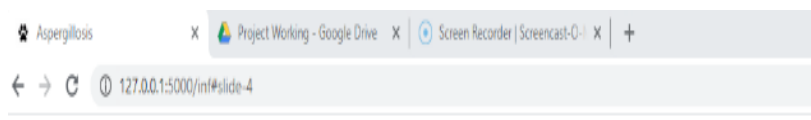

\section{Treatment}

There have been successful treatments through the administration of an antifungal drug directly into the nose or further in the nasal passage, if antifungal cat medication is prescribed, you will need to follow the full course of the medication s that symptoms do no recur.

Fig.8: Treatment of Infection shown.

\section{FLOW DIAGRAM}

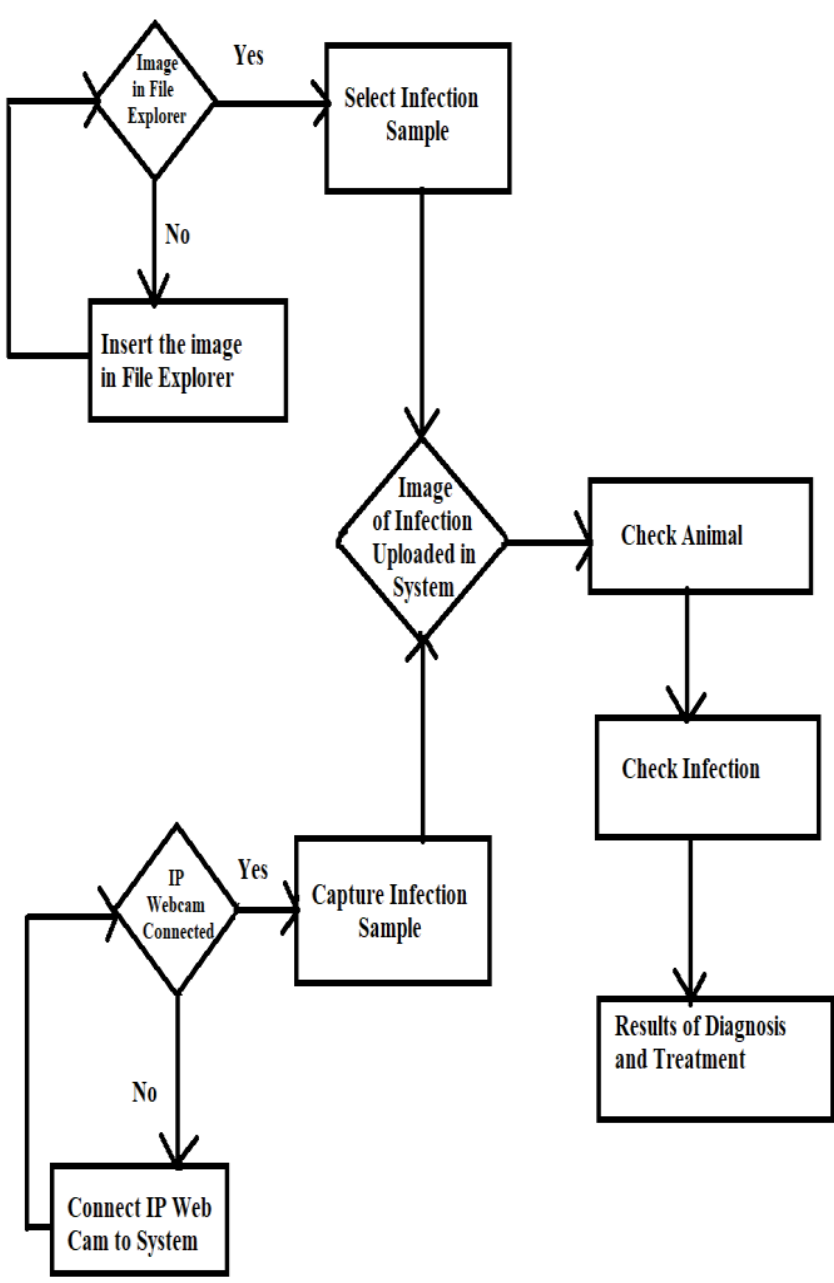

Fig. 9: Flow Diagram of Proposed System.

\section{FUTURE SCOPE}

The project has the very effective future scope, The System can be implemented in many veterinary hospitals in future for the check-ups of fungal skin infections of pet and street animals. The system will be used by many pet owners and Apartment associations in future for the treatment of small skin infections of their pets and street animals in the absence of doctors and veterinary hospitals. Also, Timely updating will be done to the system in respect of data of more and more skin diseases, also the interface. There is the scope to implement the system in app-based form in future, by this it will become more user friendly as users can use it by phones only.

\section{CONCLUSION}

So finally, we can conclude that our project is working well as it is analyzing the images of pets with infections correctly and giving the correct results about diagnosis and treatment, the overall working of project is as per desired. And the project is able to help the veterinary doctors, and the pet owners who need veterinary advice in emergency for sure in respect of treating pets' fungal infection. Also, the 
future scope of project is very nice as it cam be implemented in App based form and become more convenient in future.

\section{REFERENCES}

[1] Seyedmojtaba Seyedmousavi, Sandra de M G Bosco, Sybren de Hoog, Frank Ebel, Daniel Elad, Renata R Gomes, Ilse D Jacobsen, Henrik E Jensen, An Martel, Bernard Mignon, Frank Pasmans, Elena Piecková, Anderson Messias Rodrigues, Karuna Singh, Vania A Vicente, Gudrun Wibbelt, Nathan P Wiederhold, Jacques Guillot, "Fungal infections in animals: a patchwork of different situations", Medical Mycology, Volume 56, Issue suppl_1, 1 April 2018, Pages S165-S187

[2] Moriah Morrison, Veterinary Health Centre http://vhc.missouri.edu/small-animal-hospital/small-a nimal-internal-medicine/diseases-and-treatments/fun gal-disease//// Access on 08/05/2020.

[3] Dat Tran, ' Building a real time object recognition app with tensorflow and opencv', 22/06/2017, towardsdatascience.

[4] 'GUI Programming in Python', Python https://wiki.python.org/moin/GuiProgramming//// Access on 09/05/2020

[5] N. Singh and P. Kaur, "Comprehensive review of techniques used to detect skin lesion," 2017 2nd International Conference for Convergence in Technology (I2CT), Mumbai, 2017, pp. 100-105, doi: 10.1109/I2CT.2017.8226102.

[6] Niko Quiskamp, Sören Wildenhain, Thomas Schmidts, Peter Mayser, Frank Runkel, and Martin Fiebich: 'Image-Processing Scheme to Detect Superficial Fungal Infections of the Skin', 2015, Hindwai

[7] Keyvan Asefpour Vakilian, Jafar Massah: ', An artificial neural network approach to identify fungal diseases of cucumber (Cucumis sativus L.) plants using digital image processing', 25/03/2013, Archives of Phytopathology and Plant Protection.

[8] Yogesh Joshi: ' ' Digital Image Processing Techniques for Detecting Plant or Animal Diseases', 2015, Grin.

[9] L. Yuan, Z. Qu, Y. Zhao, H. Zhang and Q. Nian, "A convolutional neural network based on TensorFlow for face recognition," 2017 IEEE 2nd Advanced Information Technology, Electronic and Automation Control Conference (IAEAC), Chongqing, 2017, pp. 525-529, doi: 10.1109/IAEAC.2017.8054070. (Basic Book/Monograph Online Sources) J. K. Author. (year, month, day). Title (edition) [Type of medium]. Volume(issue). Available: http://www.(URL)

[10] Abhinav Datich: 'Practical Computer Vision', 02/2018, PACKT.

[11] Tahir, Muhammad. (2019). Fungus Detection Using Computer Vision and Machine Learning Techniques. Research Gate

[12] Abdellatif Abdelfattah: " Image Classification using Deep Neural Networks — A beginner friendly approach using TensorFlow', 28/07/2017, Medium.com 